\title{
SELECTIVIDAD POR EDAD Y POR SEXO DE LOS MIGRANTES EN MÉXICO, 1930-1960 *
}

\author{
Gustavo Cabrera \\ El Colegio de México
}

De los países de América Latina, México se encuentra entre aquellos que tienen poca tradición censal en cuanto a la información sobre el volumen y las características de los migrantes. De los ocho censos realizados a partir de 1895, sólo en los de 1950 y 1960 se proporcionó información sobre el lugar de nacimiento y de residencia a nivel de entidad federativa. De esta forma, cualquier intento de estimar volúmenes de migrantes en fechas anteriores a 1950, así como su distribución por edad y por sexo, requiere de la utilización de métodos indirectos.

El presente trabajo tiene por objeto examinar la selectividad según la edad y el sexo de los migrantes, en cada uno de los períodos intercensales que comprende el lapso de 1930 a 1960, referida a tres diferentes tipos de migración: migrantes entre entidades federativas, migrantes de las zonas urbanas y migrantes de la zona metropolitana de la ciudad de México.

Haciendo uso del método de "índices de sobrevivencia censales", se ha estimado para México el número de migrantes netos en cada uno de los períodos intercensales, de acuerdo con la clasificación mencionada anteriormente. Como ya es conocido, este método permite obtener, para cada sexo, estimaciones de la migración neta de la población de 10 y más años y de su distribución por grupos quinquenales de edad al final de cada decenio.

Para la interpretación de los resultados, se explican a continuación las características de las unidades geográficas establecidas:

a) Entidad federativa. México cuenta con 32 entidades federativas y las estimaciones se refieren a los intercambios de población entre ellas. Las entidades no han modificado sus límites político-administrativos desde 1930. Solamente se presentan los resultados de la migración neta para el total de las entidades.

b) Zonas urbanas. La población urbana se ha definido como aquella que vive en localidades de más de 15000 habitantes. De acuerdo con esta definición, las estimaciones se refieren a la migración que ocurrió durante los tres intervalos de tiempo en las mismas localidades que tenían más de 15000 habitantes al principio y al final de cada intervalo. En esta forma, en el período 1930-1940, la migración neta se refiere a la ocurrida en las localidades que en 1930 tenían más de

* Este artículo fue presentado como ponencia en la sesión 3 de la Conferencia Regional Latinoamericana de Población celebrada en la ciudad de México en agosto de 1970. 
15000 habitantes y precisamente las mismas en 1940. Existen otras localidades con menos de 15000 habitantes en 1930 y con más de esa cifra en 1940 que fueron excluidas en la estimación, ya que su aparición como localidades urbanas en el último año obedece a una reclasificación de su categoría rural a urbana durante el período. En los otros dos intervalos se siguió el mismo criterio.

c) Zona Metropolitana de la Ciudad de México. La delimitación geográfica de la $Z M C M$ se refiere al año de 1960 y comprende la ciudad de México y el conjunto de 12 delegaciones que forman el Dis. trito Federal, y además un número de municipios de otras entidades que colindan con el propio Distrito Federal. ${ }^{1}$

\section{SELECTIVIDAD POR EDAD}

Entidades federativas. Durante el período 1930-1940, el mayor número de migrantes masculinos perteneció al grupo de 10 a 14 años de edad, con $16.6 \%$ del total; en el segundo decenio el máximo volumen ocurrió en el grupo de 15 a 19 años, con $18.6 \%$; y en el tercer decenio fue el de 20 a 24 años, con $20.7 \%$.

Este hecho posiblemente esté en relación, por un lado, con la extensión del sistema educativo a nivel nacional, que cuando menos en el nivel primario, da lugar a que los niños y jóvenes esperen para migrar la terminación de su ciclo de enseñanza; por otro lado, un país en proceso de industrialización requiere de una mano de obra, además de mejor calificada, con edad mínima suficiente que garantice la realización de las labores que se le encomienden. También es posible que se haya efectuado un cambio tanto en el tamaño de la familia del migrante en cuanto a una tendencia de migrar de los padres que tengan un menor número de hijos, como en la composición del estado civil en donde la categoría de migrantes solteros adquiere más importancia. Estas consideraciones deberán ser corroboradas por estudios detallados sobre las características de los migrantes.

En el caso del grupo de mujeres migrantes no se presenta la situación anterior, pues acusan el mayor volumen las de 15 a 19 años de edad con ligera tendencia de aumento relativo, de $19.5 \%$ en el primer decenio a $21.7 \%$ en el último. Sin embargo, se observa que en el grupo de edad que le sigue, 20 a 24 años, hay un incremento significativo en el volumen de las mujeres migrantes, que pasan de $14.9 \%$ en el primer decenio a $19.2 \%$ en el último. De acuerdo con esto, la tendencia indica que el grupo de edad con mayor volumen posiblemente será el de 20 a 24 años, correspondiendo a un proceso semejante al que se dio en la migración masculina.

La concentración de la migración femenina en el grupo de 15 a 19 años y su estabilidad hasta 1960 pueden ser explicadas por la preferencia de la mujer por migrar hacia las ciudades de mayor tamaño del país y a emplearse en actividades características de esas edades, como las del sector terciario, en especial los servicios domésticos y otros.

La distribución de la migración en el resto de los grupos de edad

1 Véase Luis Unikel, "La urbanización y la zona metropolitana de la ciudad de México", Comercio Exterior, Vol. XVI, Núm. 11, noviembre de 1966, pp. 839-849. 
Cuadro 1

MÉxico: DistRibucrón PORCENTUAL De LA MIGRACIÓN NETA POR GRUPOS DE EDAD Y POR SEXO E ÍNDICES DE MASCULINIDAD DEL TOTAL DE LAS ENTIDADES FEDERATTVAS, $1930-1960$

\begin{tabular}{|c|c|c|c|c|c|c|}
\hline \multirow{2}{*}{$\begin{array}{l}\text { Eaad al final } \\
\text { del perfodo }\end{array}$} & \multicolumn{3}{|c|}{ Wistribucion porcentual } & \multicolumn{3}{|c|}{ Inqices de masculinioae } \\
\hline & $1930-1940$ & $1940-1950$ & $1950-1960$ & $\longdiv { 1 9 3 0 - 1 9 4 0 }$ & $1940-1950$ & $1950-196 \mathrm{C}$ \\
\hline \multicolumn{7}{|l|}{ Hombres } \\
\hline $10-14$ & 16.6 & 11.7 & 12.8 & 82 & 80 & 82 \\
\hline $15-19$ & 16.3 & 18.6 & 18.5 & 73 & 86 & 78 \\
\hline $20-24$ & 16.0 & 16.7 & 20.7 & 94 & 83 & 99 \\
\hline $25-29$ & 14.4 & 15.6 & 13.8 & 99 & 116 & 105 \\
\hline $3 c-34$ & 7.9 & 9.1 & 9.3 & 109 & 116 & 114 \\
\hline $35-39$ & 7.3 & 7.2 & 5.4 & 94 & 92 & 89 \\
\hline $40-44$ & 5.4 & 5.4 & 3.8 & 100 & 104 & 104 \\
\hline $45-49$ & 3.9 & 4.7 & 4.5 & 94 & 90 & 85 \\
\hline $50-54$ & 3.5 & 2.7 & 2.9 & 81 & 73 & 82 \\
\hline $55-59$ & 2.7 & 2.5 & 2.3 & 89 & 70 & 87 \\
\hline $60 \mathrm{y}+$ & 6.0 & 5.8 & 6.0 & 71 & 83 & 102 \\
\hline Total & 100.0 & 100.0 & 160.0 & 87 & 91 & 92 \\
\hline Volumen & 279.9 & 440.1 & 503.4 & & & \\
\hline \multicolumn{7}{|l|}{ Mujerns } \\
\hline $10-i 4$ & 17.6 & 13.2 & 14.4 & & & \\
\hline $15-19$ & 19.8 & 19.7 & 21.7 & & & \\
\hline $20-24$ & 14.9 & 18.3 & 19.2 & & & \\
\hline $25-29$ & 12.7 & 12.2 & 12.0 & & & \\
\hline $30-34$ & 6.4 & 7.1 & 7.7 & & & \\
\hline $35-39$ & 6.7 & 7.2 & 5.7 & & & \\
\hline $40-44$ & 4.7 & 4.7 & 3.2 & & & \\
\hline $45-49$ & 3.6 & 4.7 & 4.8 & & & \\
\hline $56-54$ & 3.7 & 3.4 & 3.3 & & & \\
\hline $55-59$ & 2.5 & 3.2 & 2.9 & & & \\
\hline $60 y+$ & 7.6 & 6.3 & 5.1 & & & \\
\hline Total & 100.0 & 100.0 & 100.0 & & & \\
\hline Volumen & 320.0 & 484.2 & 547.0 & & & \\
\hline
\end{tabular}

a Por 100 mujeres.

b En miles.

de ambas sexos es bastante uniforme durante los tres períodos, habiendo ligeras tendencias en algunas edades hacia la disminución y al aumento en otras.

Por otro lado, en el conjunto total de la migración neta de las entidades federativas, el mayor volumen se ha concentrado, en cada sexo, entre las edades de 10 a 29 años; representan $63.3 \%, 62.6 \%$ y $65.8 \%$ en los hombres y $64.7 \%, 63.4 \%$ y $67.3 \%$ en las mujeres, en cada uno de los tres períodos. Sin embargo, a pesar de que el mayor porciento pertenece a edades jóvenes, este no difiere considerablemente de la proporción de la población total en esas mismas edades, a diferencia de lo que ocurre en otros países. En México, en 1940, el $55 \%$ de la población masculina de más de 10 años de edad se encontraba entre 10 y 29 años de edad, frente al $63 \%$ de la población migrante. En 1960, fue de $57 \%$ en la población total y $66 \%$ en los migrantes. En Venezuela, durante 1941-1950,2 el $85 \%$ de la migración en las entidades se concentró entre 10 y 29 años mientras que este grupo

2 Juan C. Elizaga, Migraciones diferenciales en algunas regiones y ciudades de la América Latina, 1940-1950. Centro Latinoamericano de Demografía, Santiago de Chile, 1963. 
de edad constituía el $53 \%$ de la población total. En Chile, en el período $1952-1960^{3}$ de la población migrante de más de 15 años de edad, de las provenientes, el $71 \%$ pertenecía al grupo de 15 a 34 años, mientras que en la población total de más de 15 años ese grupo representó el $52 \%$.

De esta forma parece ser que en México la selectividad por edad de los migrantes es un poco menor que en otros países, en cuanto a la migración entre entidades federativas.

Zonas urbanas. El mayor volumen de la migración neta de las zonas urbanas en los grupos quinquenales de edad se concentró, para los hombres, en las edades de 15 a 19 años en los dos primeros decenios, con $26.8 \%$ y $21.8 \%$ del total, respectivamente, y en el grupo de $20-24$ con $25.2 \%$ en el período de 1950-1960. En el caso de las mujeres

Cuadro 2

MÉXICO: Distribución PORCENTUAL DE La MigRación NETA POR GRUPOS DE EDAD Y POR SEXO E ÍNDICES DE MASCULINIDAD DEL TOTAL DE LAS ZONAS URBANAS DEL PAfS, $1930-1960$

\begin{tabular}{|c|c|c|c|c|c|c|}
\hline \multirow{2}{*}{$\begin{array}{l}\text { Edad al final } \\
\text { del porfodo }\end{array}$} & \multicolumn{3}{|c|}{ Wistribucion porcentual } & \multicolumn{3}{|c|}{ Inaices de masculiniuad } \\
\hline & $1930-1940$ & $1940-1950$ & $1950-1960$ & $1930-1940$ & $1940-1950$ & $1950-1960$ \\
\hline \multicolumn{7}{|l|}{$\underline{\text { Hombreg }}$} \\
\hline $10-14$ & 24.3 & 15.2 & 14.0 & 70 & 68 & 73 \\
\hline $15-19$ & 26.8 & 21.8 & 24.3 & 54 & 70 & 70 \\
\hline $20-24$ & 24.9 & 17.3 & 25.2 & 67 & 66 & 89 \\
\hline $25-29$ & 16.4 & 14.8 & 10.8 & 101 & 115 & 104 \\
\hline $30-34$ & 0.4 & 7.9 & 8.6 & 10 & 121 & 144 \\
\hline $35-39$ & 0.5 & 6.7 & 3.0 & 5 & 81 & 72 \\
\hline $40-44$ & $-\mathrm{b} /$ & 4.8 & 2.8 & - & 83 & 100 \\
\hline $45-49$ & $1 . \overline{8}$ & 4.6 & 4.3 & 62 & 78 & 10 \\
\hline $50-54$ & 3.4 & 2.0 & 3.0 & 53 & 44 & 75 \\
\hline $55-59$ & 1.5 & 2.6 & 2.4 & 43 & 67 & 77 \\
\hline $60 y+$ & $-b /$ & 2.3 & 7.6 & - & 39 & 41 \\
\hline Total & 100.0 & 100.0 & 100.0 & 54 & 75 & 83 \\
\hline Volumen & 121.0 & 378.0 & 367.1 & & & \\
\hline \multicolumn{7}{|l|}{ Kujeres } \\
\hline $10-14$ & & 16.8 & 16.0 & & & \\
\hline $\begin{array}{r}15-19 \\
19\end{array}$ & 27.7 & 23.5 & 28.8 & & & \\
\hline $20-24$ & 20.6 & 19.7 & 23.5 & & & \\
\hline $25-29$ & 9.0 & 9.7 & 8.6 & & & \\
\hline $30-34$ & 2.3 & 4.9 & 5.0 & & & \\
\hline $35-39$ & 5.5 & 6.1 & 3.4 & & & \\
\hline $40-44$ & 4.3 & 4.3 & 2.3 & & & \\
\hline $45-49$ & 1.6 & 4.3 & 3.2 & & & \\
\hline $\begin{array}{l}50-54 \\
55-50\end{array}$ & 3.5 & 3.4 & 3.3 & & & \\
\hline $\begin{array}{l}55-59 \\
60 y+\end{array}$ & $\begin{array}{l}1.9 \\
4.2\end{array}$ & $\begin{array}{l}2.9 \\
4.4\end{array}$ & 2.6 & & & \\
\hline Total & 100.0 & 100.0 & 100.0 & & & \\
\hline Yolumen of & 2358 & 5030 & 149? & & & \\
\hline & 225.0 & 303.9 & 442.0 & & & \\
\hline
\end{tabular}

a Por 100 mujeres.

b Valores negativos.

c En miles.

3 Arthur M. Conning, Estimaciones de la migración interna neta, clasificada por edad y por sexo, en las provincias $y$ regiones de Chile durante los años de 1930-1940, 1940-1952 y 1952-1960. Centro Latinoamericano de Demografía, Santiago de Chile. 
la mayor concentración se realizó en las edades de 15 a 19 años en los tres períodos, con $27.7 \%, 23.5 \%$ y $28.8 \%$ del total, respectivamente.

Las cifras relativas anteriores son considerablemente mayores que las observadas en el caso de la migración neta de las entidades, denotando una mayor selectividad por edad de los migrantes urbanos. Esto último puede apreciarse mejor con la concentración de los migrantes netos entre las edades de 10 a 29 años de edad; en el caso de los migrantes hombres se observa que el $92 \%$ del total perteneció a ese grupo de edades en el período $1930-1940$ y en los dos siguientes períodos disminuye la concentración a $69 \%$ y $74 \%$, respectivamente. En las mujeres no hay variaciones si se comparan los intervalos extremos en donde cerca del $77 \%$ se concentró en las edades de 10 a 29 años. Sin embargo, hay una disminución al $70 \%$ en el período intermedio.

\section{Cuadro 3}

Ciudad de México (Zona Metropolitana): Distribución porcentual DE LA MIGRACIÓN NETA POR GRUPOS DE EDAD Y POR SEXO E ÍNDICES DE MASCULINIDAD, 1930-1960

\begin{tabular}{|c|c|c|c|c|c|c|}
\hline \multirow{2}{*}{$\begin{array}{l}\text { Edad al tinal } \\
\text { del porlodo }\end{array}$} & \multicolumn{3}{|c|}{ Listribucion poroentual } & \multicolumn{3}{|c|}{ Inaices de masculinicad } \\
\hline & $.1930-1940$ & $1940-1950$ & $1950-1960$ & $1930-1940$ & $1940-1950$ & $1950-1950$ \\
\hline \multicolumn{7}{|l|}{ Hombres } \\
\hline $\begin{array}{l}10-14 \\
15-19 \\
20-24 \\
25-29 \\
30-34 \\
35-39 \\
40-44 \\
45-49 \\
50-54 \\
55-60 \\
60 y+\end{array}$ & $\begin{array}{r}14.5 \\
17.8 \\
20.7 \\
18.7 \\
9.4 \\
6.7 \\
4.8 \\
2.1 \\
2.5 \\
1.4 \\
1.4\end{array}$ & $\begin{array}{r}11.0 \\
19.1 \\
18.9 \\
19.3 \\
10.1 \\
7.2 \\
4.7 \\
3.9 \\
2.0 \\
2.2 \\
1.6\end{array}$ & $\begin{array}{r}10.0 \\
20.7 \\
26.5 \\
16.5 \\
11.6 \\
4.3 \\
2.3 \\
3.3 \\
1.9 \\
1.9 \\
1.0\end{array}$ & $\begin{array}{r}74 \\
56 \\
73 \\
90 \\
117 \\
80 \\
85 \\
71 \\
79 \\
49 \\
47 \\
21\end{array}$ & $\begin{array}{r}70 \\
71 \\
71 \\
117 \\
116 \\
89 \\
86 \\
75 \\
49 \\
58 \\
28\end{array}$ & $\begin{array}{r}67 \\
70 \\
92 \\
104 \\
121 \\
81 \\
95 \\
90 \\
71 \\
72 \\
33\end{array}$ \\
\hline Motal. & 100.0 & 100.0 & 100.0 & 71 & 78 & 83 \\
\hline Volumen $\mathrm{n}^{\mathrm{b}}$ & 121.9 & 261.2 & 301.6 & & & \\
\hline \multicolumn{7}{|l|}{ Mujeres } \\
\hline $\begin{array}{l}10-14 \\
15-19 \\
20-24 \\
25-29 \\
30-34 \\
35-39 \\
40-44 \\
45-49 \\
50-54 \\
55-59 \\
60 y+\end{array}$ & $\begin{array}{r}14.0 \\
22.1 \\
19.8 \\
14.3 \\
5.4 \\
6.0 \\
4.1 \\
2.4 \\
4.0 \\
2.4 \\
5.5\end{array}$ & $\begin{array}{r}12.4 \\
20.8 \\
20.8 \\
12.7 \\
6.5 \\
6.4 \\
4.3 \\
4.3 \\
3.5 \\
3.2 \\
5.0\end{array}$ & $\begin{array}{r}13.0 \\
24.3 \\
23.8 \\
13.0 \\
7.7 \\
4.8 \\
2.2 \\
3.1 \\
2.5 \\
2.5 \\
3.1\end{array}$ & & & \\
\hline Total & 100.0 & 100.0 & 100.0 & & & \\
\hline Vol umen $\mathrm{b}$ & 176.4 & 338.9 & 368.8 & & & \\
\hline
\end{tabular}

n Por 100 mujeres.

b En miles.

A pesar de las pocas observaciones y elementos de que se dispone, la regularidad que se presenta en ambos sexos en el sentido de que en el período 1940-1950 disminuye la concentración de los migrantes en las edades de 10 a 29 años, situación que se da también en los 
migrantes de las entidades federativas, aunque menos marcada, parece señalar que hay una relación inversa entre el incremento en los valores absolutos de la migración y su selectividad por edad. Es decir, que mientras mayor es el aumento de la migración en cifras absolutas, menos se concentran los migrantes en determinados grupos de edad. Examinando nuevamente las cifras anteriores, el volumen mínimo de migrantes se dio en el sexo masculino en 1930-1940 con 121000 y en ese período es cuando se produjo la máxima concentración de $92 \%$. En ese mismo período, las 226000 mujeres migrantes casi duplicaban la cifra de los hombres, siendo su concentración de $77 \%$ en las mismas edades. En el período intermedio, 1940-1950, se incrementó en forma notable la migración de ambos sexos y la concentración disminuyó especialmente en los hombres a $69 \%$. Por último, al disminuir el volumen de los migrantes en 1950-1960, se elevó nuevamente su concentración en ambos sexos a $74 \%$ en los hombres y a $77 \%$ las mujeres. Por supuesto que el volumen sólo puede ser uno de los elementos que influyen en la selectividad de los migrantes por edad.

Zona metropolitana de la Ciudad de México. El mayor volumen de los migrantes hombres de la $Z M C M$ se concentró en las edades de 20 a 24 años en $1930-1940$ y en $1950-1960$ con $20.7 \%$ y $26.5 \%$, respectivamente. En el período intermedio, el mayor volumen correspondió al grupo de 25 a 29 años con $19.3 \%$, ligeramente superior al grupo 20-24. años con el $18.9 \%$. Esta concentración no difiere de la observada en las zonas urbanas.

La concentración en el grupo de 10 a 29 años tampoco difiere notablemente del caso anterior y su comportamiento en los tres períodos es semejante al observado en los migrantes de las entidades federativas y de las zonas urbanas. Para el sexo masculino la concentración representó el $71.7 \%, 68.3 \%$ y $73.7 \%$, respectivamente, en cada período y para el sexo femenino el $70.2 \%, 66.7 \%$ y $74.1 \%$, del total de migrantes.

Como podría esperarse, la selectividad de los migrantes por edad de la $Z M C M$ es muy parecida a la de las zonas urbanas, ya que gran parte de la corriente migratoria rural-urbana se dirige hacia la metrópoli.

Aquí se observa también que cuando se dan los mayores incrementos en la migración, período 1940-1950, disminuye su concentración en el grupo 10 a 29 años, es decir, que la selectividad por edad disminuye.

\section{Selectividad POR SEXo}

La selectividad por sexo de los migrantes, representada por el índice de masculinidad, revela una mayor migración del sexo femenino, especialmente en las zonas urbanas y la $Z M C M$, con una tendencia al equilibrio de los migrantes por sexo durante los tres períodos. Del total de migrantes de las entidades, el índice de masculinidad fue de 87 hombres por cada 100 mujeres en 1930-1940, de 91 en el siguiente decenio y de 92 en el último. El de las zonas urbanas se transformó de 54 hombres por 100 mujeres a 75 y 83, respectivamente para cada intervalo. Por último, el índice de los migrantes de la $Z M C M$ fue de 71, 78 y 83 en cada período. Esto revela que en México la selectividad 
por sexo de los migrantes ha disminuido notablemente en el caso de la migración rural-urbana, con menos intensidad en la $Z M C M$ y ligeramente en la migración entre entidades federativas, si bien en el último período se aproximan los valores del índice en los tres tipos de migración, siendo iguales en los casos de las zonas urbanas y la ZMCM.

Por edad, la selectividad de la mujer es notable en aquellos grupos donde se concentra la mayor parte de los migrantes, especialmente en el de 10 a 19 años. La mayor selectividad en esas edades corresponde al período 1930-1940, siendo superior para las zonas urbanas y la $Z M C M$ con índices de masculinidad del orden de 55 para el grupo de 15 a 19 años.

En el grupo de 20 a 24 años de edad principia un equilibrio entre los sexos, pasando a ser superior el número de hombres migrantes en las edades de 25 a 29 años y de 30 a 34 años.

De esta manera, la mayor selectividad femenina en el total de migrantes se debe básicamente a la que se da entre las edades de 10 a 19 años. El comportamiento por edad de la proporción entre mujeres y hombres migrantes obedece, entre otras causas, a la diferente atracción que ejercen diversos tipos de ocupación propias de determinadas edades y sexos. 